\title{
Index of difficulty which is an effective factor on especial skill formation in basketball free throw: index of learning difficulty hypothesis
}

\author{
Roohollah Talebi ${ }^{\mathrm{ABCDE}}$, Hamid Reza Taheri ${ }^{\mathrm{ABCDE}}$, Mahdi Sohrabi ${ }^{\mathrm{ABCDE}}$ \\ Physical Education Faculty and Sports Sciences, Ferdowsi University of Mashhad, Iran
}

Authors' Contribution: A - Study design; B - Data collection; C - Statistical analysis; D - Manuscript Preparation; E - Funds Collection.

\begin{tabular}{|c|c|}
\hline \multicolumn{2}{|l|}{ Abstract } \\
\hline Purpose: & $\begin{array}{l}\text { he purpose of this study is to examine the specific memory representation in special skill of Basketball Free } \\
\text { hrow in distances (a"set shot") except } 4.5 \mathrm{~m} \text { distance. It is aimed to examine the role of the Index of Difficulty } \\
\text { D) in creating the specific memory in other distances except the distance of penalty shoots. }\end{array}$ \\
\hline Material: & $\begin{array}{l}\text { Fifteen skilled basketball players ( } \mathrm{M} \text { age }=21.6 \text { years, SD }=2.45 \text { years) were chosen from sixty players; they } \\
\text { performed } 70 \text { trials from } 7 \text { different distances on the first day ( } 10 \text { SHOTS from each distance toward the } \\
\text { basket). The next day, each player performed } 10 \text { SHOTS from each distance, but this time, it was different in } \\
\text { terms of the baskets' sizes and their quantities. }\end{array}$ \\
\hline Results: & $\begin{array}{l}\text { he result of linear regression on trials score on the first day revealed the representation of special skill in } \\
\text { enalty point and on the second day, representation of special skill was seen in } 3 \text { different distances from } \\
\text { e ring (manipulated ring). These results indicate that Index of Difficulty protection can be a factor of private } \\
\text { eemory representation in distances except } 4.5 \mathrm{~m} \text { distance. }\end{array}$ \\
\hline Conclusions: & $\begin{array}{l}\text { Results suggest that mass training stabilizes the ratio of target distance and width (Index of Difficulty) in mind } \\
\text { and this ratio is the main reason of specific memory representation in basketball free throw. }\end{array}$ \\
\hline Keyw & \\
\hline
\end{tabular}

\section{Introduction}

One of the important challenges in motor learning is the way of learning and conditions of motor memory representations [1]. Some researchers emphasized on the generality of learning and believe that the learned motors have the capacity to be parameterized and be performed in various environmental conditions and even with other organs [2, 3]. In contrast with this theory, some scientists believe on the specificity of motor skills learning. In other words, movements will be specified according to their learning conditions and followed by dedicated memory representation [4]. Recent studies about darts, baseball, and basketball skills support these kinds of theories, in which the increase in distance will decrease the performance and skillful persons have special performance in a single distance [5-8]. An especial skill is when the performance of a single action from within a class of actions produces an advantage in performance. This advantage is in a single action over others in the same class of actions and is said to result from massive amounts of practice in performing the specific action [9]. An especial skill effect was first demonstrated by Keetch et al. [9], where in highly skilled basketball players executing the set shot from the FT $(15 \mathrm{ft}[4.57 \mathrm{~m}]$ from the basket) were found to be more accurate than at closer distances as predicted by a single linear regression equation. Keetch et al. [9] attributed the uniqueness of an especial skill to the accumulation of large amounts of practice at a specific distance. Significantly, these experiments led the authors to suggest two features about the memory representation underlying the set-shot skill, that the set-

\footnotetext{
(C) Roohollah Talebi, Hamid Reza Taheri, Mahdi Sohrabi, 2018
} doi:10.15561/20755279.2018.0205 shot representation was sufficiently general as to allow set SHOTS to be taken from many different distances on the court and that the representation had a specific component such that an advantage in outcome accuracy was obtained whenever a set shot was attempted from one specific distance of $15 \mathrm{ft}$ from the basket, the distance at which massive amounts of practice had been performed. Several explanations have been proposed describing the contents of the memory representation underpinning the especial skill effect [10], however, it remains unknown at what point the development of the especial skill emerges in a basketball player.

These results distinguish motor learning and memory representation in that this distance from the other distances is in contrast with the beliefs of theoreticians about the generality of motor memory and general motor learning [11]. Adams attempted to present a model with the name closed-loop to show that the findings related to the specificity of learning a movement consist of background and environmental information. He said that perceptual trace is responsible for learning and memory representation that is performed very specifically. A new view about motor control and learning is proposed which is in contrast with the basis of the motor program. This attitude which is called dynamic system perspective looks at the motor system of human from the perspective of being dynamic and non-linear [12]. From the perspective of this view, the state of steadiness in dynamic condition is called stability. It will start running when a perturbation and disorder occurs in the system and it will lead the system to a steady state [12-15]. By adjusting the condition for establishing special skill and performing it in various distances, it points to the fact that throw in different distances creates 
a kind of disorder. This instability will be stable at the standard distance of especial skill throw. In this regard, experienced players throws toward targets follow a nonlinear pattern. The special skills will be closed from unstable state to steady state. In the views of researchers, establishing a particular skill requires frequent training in that distance. Training a special skill such as free throw follows Fitts's Law as a one way targeting skill that the time and accuracy of movement are dependent on the distance and its change. At the time of learning, a motor plan is created in mind that has the specific time and space scale of that task. Hacker [16] said that the ratio between the size and accuracy of movement can be examined. He considered it as an important and main nature in motor control and transition of learning. Nowadays, this issue is studied with the concept of index of difficulty. It can be said that the result of movement is dependent on the ratio of size and distance from the target. This ratio will remain fixed when the accuracy and size are changed together at the time of performing a movement [15]. Although this consistency does not directly refer to a common strategy, Hacker's results indicated that the ratio between size and accuracy and also the form of movement must be considered as an important feature of motor control and transition [16]. He also reported that this law is true about various targeting movements and the involved muscles play no role in that [17]. According to Thelen et al. [14], the form of movement which is defined by the ratio of size and accuracy in 1-D space determines the time of movement. Taheri et al. [15] examined the effect of parameter change on the result and performed a jump shot of basketball. Results showed that the accuracy of throw decreased as a function of task limitations. The angle of release of ball decreased due to the increase of distance. The speed of release of ball is increased by the increase of distance. Also, Kwon et al. [17] examined the role of the ratio between the accuracy and size of movement (ID) in transfer learning. Transfer of skilled movement is better when both the size and accuracy of movement are changed by the same factor (ID is constant) than when only size or accuracy is changed. They infer that the size-accuracy ratio captures the control strategies employed during practice and thus promotes efficient transfer. Berslin et al. [18] in a survey examined the representation of a special skill and perceived that a special skill can be established even in short term. The type of training can be one of the effective factors of the formation of dedicated memory and increase of special skill. Newell [19] and Stoeckel [20] examined the visual context effect on performing and learning free throw skill. The results revealed that one of the conditions of establishing a special skill and showing it in free throw performance is trained visual context. It is possible that during training, a ratio distance-accuracy (difficulty index) task takes form in the mind of the learner. This task and visual context and parameter can establish a special skill in learning which will validate the dedicated memory hypothesis.

Hypothesis and Purpose: Now the question is whether adjustment of index of difficulty is an effective factor in motor learning for dedicated memory representation? And can we create the performance of a skillful person in special skill in other distances by preserving task difficulty?

\section{Material and Methods}

Participants: Fifteen male basketball players were chosen from 60 Master League point guards ( $\mathrm{M}$ age = 21.6 years, $\mathrm{SD}=2.45$ years) from Iran basketball national team and they partook in the experiment. All of them were Shooting guard and were selected purposefully, their height and weight were important in their selection and homogeneity of the group.

Research Design: All participants gave written informed consent to participate in the experiment, ethical approval was granted by the lead institution. For conducting the test, number 7 standard ball of basketball made by Molten Company, standard basket and backboard of basketball and a basket size by 53 and $39 \mathrm{~cm}$ were used. In this experiment, 15 people participate in one group. They were requested to perform their SHOTS with the standard ball (size 7 of Molten company) based on the regulations of FIBA of a standard basket which is located at the distance of $3.05 \mathrm{~m}$ from the ground. They were instructed not to separate their foot from the ground during the SHOTS (do not jump). The procedure was in this form that the group performs the SHOTS from 7 different distances on a direct line exactly in the face of the basket. According to the figure, 7 distances have been determined by white adhesive tape $(2 \times 5 \mathrm{~cm}), 3$ distances were close to the basket and less than $4.5 \mathrm{~m}$ (free throw standard distance) and 3 distances were more than $4.5 \mathrm{~m}$. In these distances, the difficulty index of each distance was different because the size of the basket is stable and the distance is changing. For determining the distances domain of SHOTS, we referred to the studies of Taheri et al. [15] in which non-change of a motor pattern of free throw consists of not separating the subjects foot from the ground and using one hand throw. The desired distance was determined by that.

After warm up, all the participants performed 10 SHOTS from each distance toward the basket. The participants rested for 5 minutes between each 10 SHOTS of a block and after that they went to perform the next block. After the performance of participants in this mission, the next day, they performed 10 SHOTS from each distance, but this time, it was different in terms of the size and number of the baskets. This time, two baskets were used, one of the baskets was more than the standard size (53 cm diameter) and the other was smaller than the standard basket (39 $\mathrm{cm}$ diameter) and the SHOTS were performed in the distances of 2 and 6 toward the baskets. In the distances of 2 and 6, with the change of the width of the basket, the index of difficulty of throw was similar to the FT. The participants just like the previous day rested for 5 minutes after performing the 10 shot of a block, they again went to the next block in the next distance. Conduction of the experiment was in an individual form and three examiners were used. The first examiner gives 
necessary instructions to the performers, the second examiner records the scores and the third examiner delivers the ball to the subject immediately after each shot (18). Each participant performs 10 SHOTS for warming up and then releases three SHOTS from each distance toward the basket. The SHOTS number was totally 70 SHOTS per day [9]. Task performance was assessed using a 2-point scoring system in which each shot was registered as hit or miss according to official basketball rules. For each trial, mean score of SHOTS were calculated across the 10 trials from each position.

Statistical Analysis: The statistical method of this study is ANOVA, linear regression on the score of the group in each distance and paired t-test. ANOVA was used to determine the difference in scores of the group in different distances and to determine the place of these differences. Pearson's correlational statistic was used to examine the relation between distance and throwing performance.
To test specifically for the presence of the especial skill, individual linear regressions were calculated on the basis of the four positions other than the actual FT position at $4.5 \mathrm{~m}$ to generate predicted values for the FT distance (see Keetch et al. [9], for this procedure). The predicted values for the FT distance were then compared with the actual percentage of SHOTS from that position. A t-test was used to compare all throws of this two day. All the statistical analyses were done by Excel and SPSS 20.

\section{Results}

Actual mean score of SHOTS at each of the seven positions as well as the predicted values for the FT position at $4.5 \mathrm{~m}$ are presented in Figure 2 for two days. The mean score of SHOTS decreased with increasing distance in all positions in the first day (except FT), which was supported by negative linear correlations (all rs $<-0.24$, ps $<0.001)$ and a significant distance effect, $t(9,132)=$

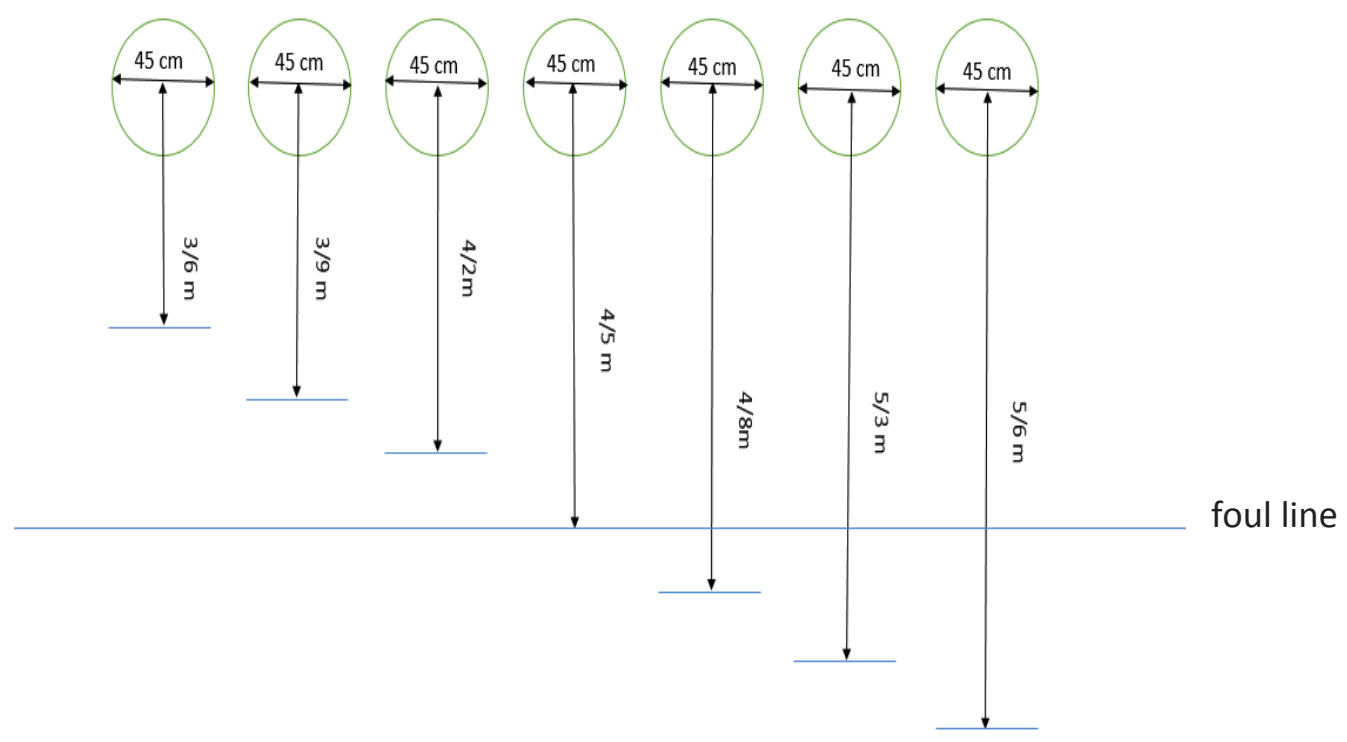

Figure 1: SHOTS from various distances toward the standard basket by subjects

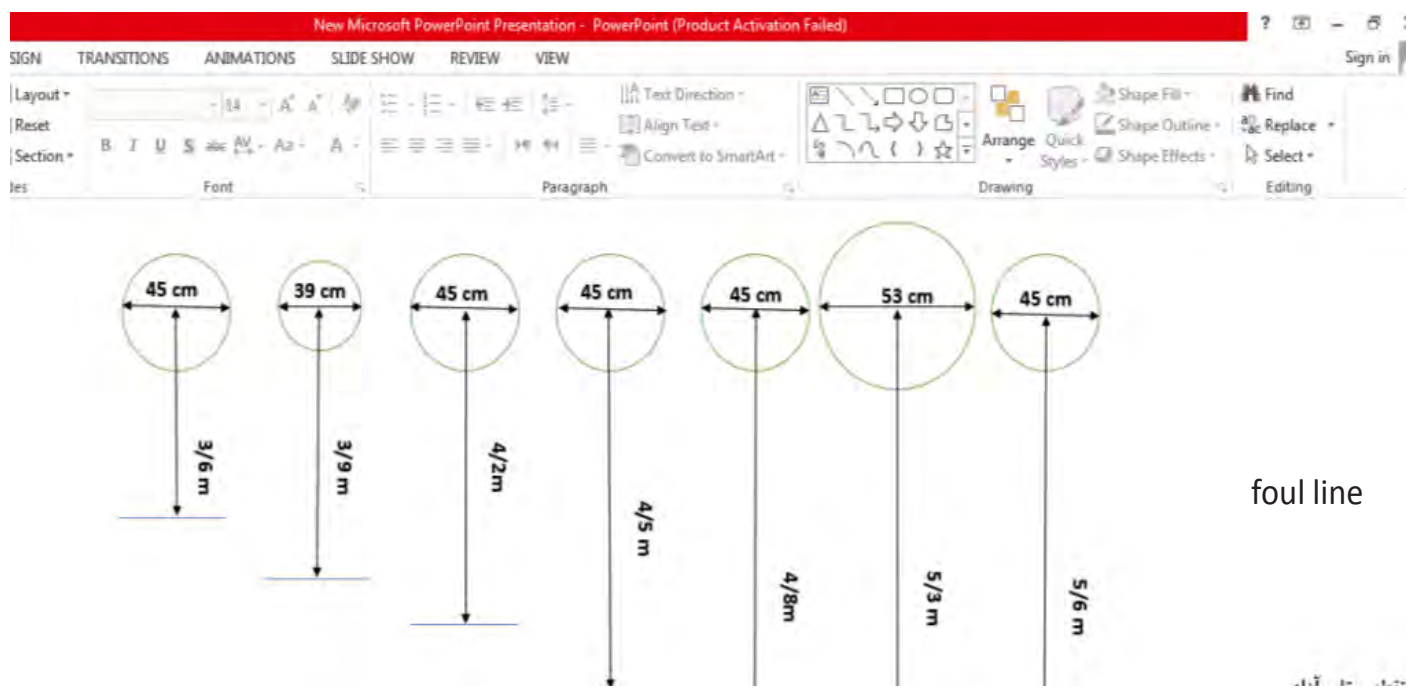

Figure 2: SHOTS from various distances during the manipulation of second and sixth distances by the subjects. 
$-6.551, \mathrm{p}<.001, \eta_{\mathrm{p}}^{2}=.894$ and the mean score of SHOTS decreased with increasing distance in all positions in the second day (except 3 distance), which was supported by negative linear correlations (all rs $<-0.24$, ps $<0.001$ ) and a significant distance effect, $\mathrm{t}(9,132)=-6.551, \mathrm{p}<$ $.001, \eta^{2}=.894$. An analysis of variance was run on the performance scores for the six nonfuel line and fuel line locations to standard ring. Normality and equality of dependent variable variance were examined by Leven's test $\left(\mathrm{f}_{(63,6)}=0.281\right.$ and $\left.\mathrm{P}=0.944\right)$. The results of ANOVA on the scores of free throw from different distances toward the basket showed a significant difference between the scores of the group in SHOTS from different distances ( $\mathrm{f}$ $(63,6)=85.745$ and $\mathrm{P}=00.0)$. According to the Tukey post hoc test, the place of all these differences between all states can be seen except 3.9 with 4.2 and also FT with 3.6 and 3.9. Also, an analysis of variance was run on the performance scores for the six nonfuel line and fuel line locations to ring with different size. Normality and equality of dependent variable variance were examined by Leven's test ( ${ }_{(63,6)}=0.66$ and $\mathrm{P}=0.999$ ). The results of ANOVA on the free throw toward the basket with different sizes showed that there is a significant difference between all states except FT and $3.9 \mathrm{~m}$ distance- FT and $5.3 \mathrm{~m}-3.9$ and $5.3 \mathrm{~m}$. By examining the average scores of groups, it was determined that no significant difference among these distances showed the same performance of subjects in these distances (3.9, 4.5, and 5.3), although this lack of difference was not observed in the state of throwing from different distances toward the standard basket. Paired t-test was used to compare the performance of the group in the state of throwing from a determined distance toward standard and manipulated basket. The performance of group from the distances of 3.9 and $5.3 \mathrm{~m}$ toward standard and manipulated basket was compared. The results showed that there is a significant difference in subjects' performance in $5.3 \mathrm{~m}$.

The linear regression results and the $\mathrm{R} 2$ value in the first test of a shot from different distances toward standard basket showed that $89.4 \%$ of the variance of performance is determined by the distance from the basket. Linear regression coefficient from the basket on the performance $(=ß-0.946)$ based on the value of $t$ test $(-6.551)$ is significant (0.001). It showed that the distance from the target can predict the performance negatively. Hence, the increase in distance from target reduces the performance. According to the calculated line equation for the first test ( $\mathrm{Y}=1.76-0.240 \mathrm{x})$ and the value of the gradient of a line, we can say that by increasing the distance from the basket by $30 \mathrm{~cm}$, their performance is decreased by about 0.072 scores on the average. The difference of real performance of subjects in comparison with predicted score by regression equation in a shot toward standard basket showed the establishment of a special performance at the distance of each throw. The results of a linear regression on the scores of SHOTS toward manipulated baskets on the second day revealed that $57.4 \%$ of performance variance is determined by the distance from the basket.

Table of paired t-test comparison of the performance of group's free throw in different conditions of distance and width of target

\begin{tabular}{llllll}
\hline $\begin{array}{l}\text { Condition of } \\
\text { throw }\end{array}$ & Average & Standard deviation & $\mathbf{T}$ & Degrees of freedom & $\begin{array}{l}\text { Level of } \\
\text { significant }\end{array}$ \\
\hline B-B' & -0.040 & 0.0966 & -1.309 & 9 & 0.223 \\
D-D' & 0.030 & 0.0949 & 1.00 & 9 & 0.343 \\
F-F' & -0.300 & 0.0471 & -20.125 & 9 & 0.00 \\
\hline
\end{tabular}

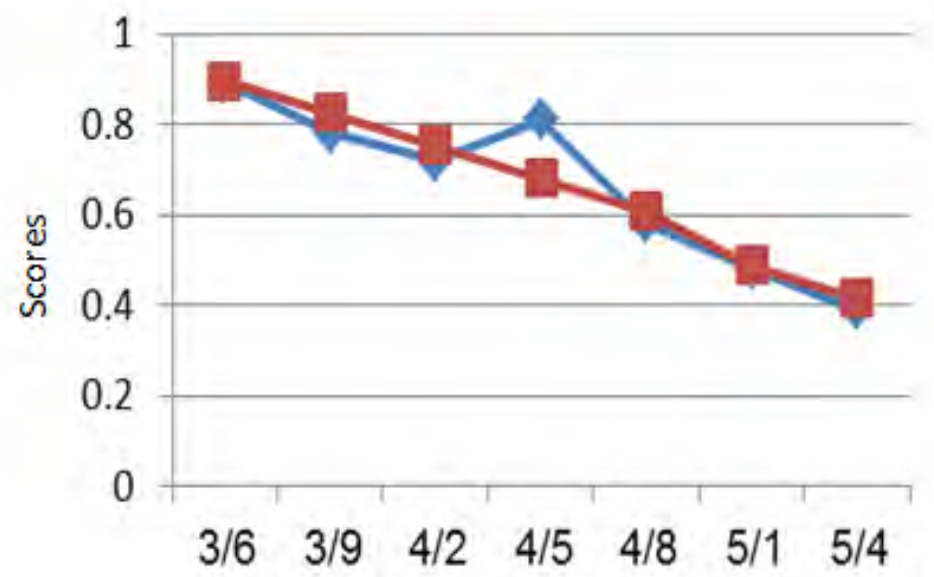

distance

Figure 3. Actual success rates (blue line) from each of the seven positions for the group and predicted values based on regression analysis are displayed (red line) on the first day. 


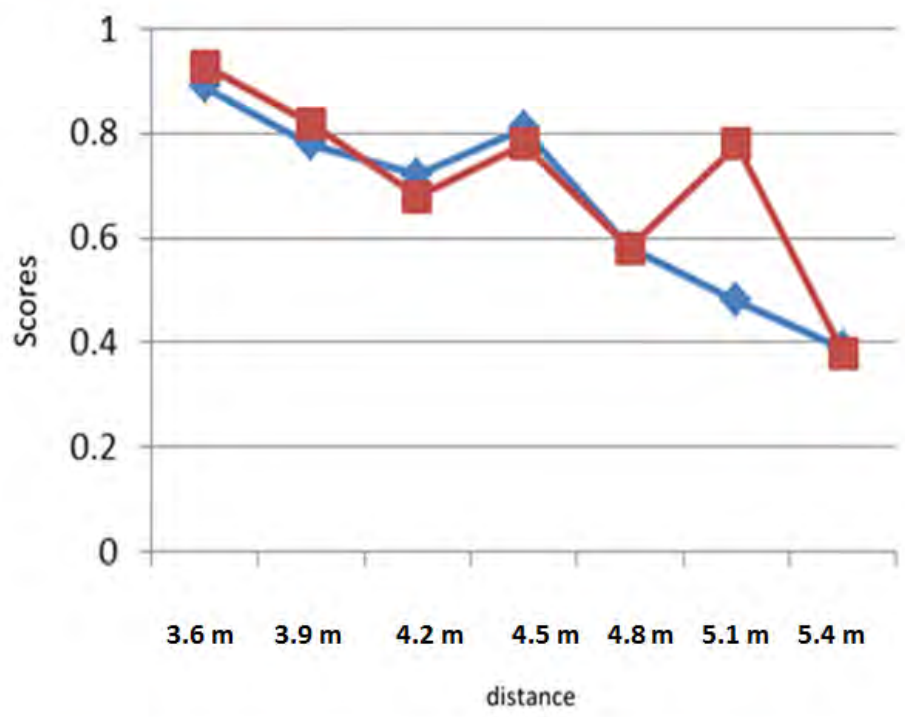

Figure 4. Actual success rates (blue line) from each of the seven positions for the group and predicted values based on regression analysis are displayed (red line) on the second day.

The regression coefficient of distance from the basket on the performance of $ß=-0.519$ according to the value of $\mathrm{t}$-test $(\mathrm{t}=-2.601)$ is significant (0.048). It showed that the distance from the target can predict the performance negatively. Therefore, the increase in distance from target causes decrease in the performance. According to the numerical value of R2, there is a low correlation between the two variables. The calculated line equation ( $\mathrm{Y}=1.568$ $0.189 \mathrm{x})$ indicated that by increasing the distance from the basket by $30 \mathrm{~cm}$, their performance is decreased by about 0.058 scores on the average (according to the negative sign of gradient). The real scores of the group in the second test (shot toward baskets with different size) were different from the predicted scores by regression equation at the distance of $5.3 \mathrm{~m}$. This difference indicates better performance of the subjects than the predicted performance in that distance.

\section{Discussion}

Results of the present study regarding free throw of 15 skillful players' performance toward standard and manipulated (width of the target is changed) basket from 7 distances showed that just like many studies, the performance of players follows the line equation except at the FT in which it will be decreased by the distance from the target. By considering the fact that the width of the target was increased and the difficulty index was the same as FT, the results revealed that the performance of each person at a distance except free throw line violates from the predictive line equation of performance and has a significant difference with their shot performance from that distance to the standard basket. In general, the results showed the lack of significant difference between the performance of group at the distances of 2, 4 and 6 (same index of difficulty).

A sport skill performed by highly trained athletes provides a clear demonstration of both the specific and general capabilities of exceptional human motor performance. Research has demonstrated that the basketball shot is one of such sport skill. This shot is almost always practiced from a line that is 15 feet from a point directly beneath the basket (i.e., a free throw from the FT). The set shot is rarely practiced from other locations because of its limited usefulness in game play. The finding that a highly specific exemplar (the free throw) could exist amid the background of a very general performance capability (set SHOTS), which we termed an especial skill, poses a real and significant problem for theories of motor control, including Schema theory [1]. The existence of an especial skill (the foul shot) is problematic because according to the theory, the foul shot should belong to a class of motor skills (set SHOTS) that becomes increasingly generalized with advanced skill. These findings suggest that years of practice at the FT produce a skill that has a specific motor control advantage at that particular distance that provides little or no detectable advantage for any other distance, irrespective of its proximity to the FT [9]. One interpretation in terms of Schema theory is that the foul shot from the free throw line is represented by one specific, exceptionally well-learned motor program. A separate GMP is developed and represents the set SHOTS from the non- FT locations [19]. The evidence for the existence of especial skills would be weakened if it were found only to exist for one class of motor skills (basketball set SHOTS). In the study that we presented here, two alternative explanations for the existence of especial skills were examined. One possibility is that massive amounts of practice at one specific instance within a class of skills improve the parameter-specification process for that unique instance. We call this the learned parameters hypothesis. An alternative suggestion is that embedded within the learned representation for the free throw and is a unique visual context for the performance of that particular set shot (which we term the visual-context hypothesis). Breslin [10] showed that special skill can be established by 300 SHOTS and it may not require mass 
training. Studies showed that a motor program can create some movements for a special movement in a category. Formation of dedicated memory and representation of that is dependent on the kind and conditions of training.

The findings suggest that visual context information influences the presence of specificity effects in experienced performers. The findings have theoretical implications for explaining the memory representation underlying the especial skill effect in basketball [20]. Of course, a key issue here is the definition of what a "condition" is, and how the effect of changing these conditions would affect the performance of motor skill. One attempt has classified these specificity effects as sensory-motor specificity, context specificity, or processing specificity [1]. An example of altered sensory-motor conditions is seen in experiments in which sensory information (e.g., vision) is systematically added or removed at the time of test [21, 22]. These experiments have shown that either removing or adding sensory information, and thus changing the information that had been available during the learning trials, had a degrading effect on the performance of the test trials. Additionally, data revealed that the degrading effect was more pronounced as the number of practice trials increased [23].

It can be said that training of a skill causes memory consolidation of that movement and creates an image of the size of the target and distance from the target and environmental condition in our memory. Perhaps, the index of difficulty of the task can be a sign for representation of this image in the person's mind. When the index of difficulty is preserved, the performance will be preserved too. This study assumed that one of the reasons of creating especial skill at an especial distance is the consolidation and effect of index of difficulty and the ratio of distance to the width of the target. The human brain uses it as a factor for creating motor adjustments and motor command. However, in spite of the frequent demonstration of specificity in motor control and learning, there have been few attempts to model these effects in theories. One exception was Adams [4] closedloop theory, in which he represented the accumulation of skill as the learning of a specific representation in memory. According to Adams's theory, the learning of a blindfolded positioning movement resulted from the strengthening of memory of a specific underlying neural representation, which he termed the perceptual trace. We have shown that those with experience of basketball free-throw shooting will demonstrate the especial skill effect but instead, their performance will not follow a linear pattern where in performance outcome decreases as distance from the basket increases in the second experiment. We have also shown that players developed an especial skill demonstrated by a less linear type trend in shooting performance across distance. We presented two ways of analyzing and explaining the presence of the especial skill effect. The first uses two straight lines as regression functions. The second employs a nonliner model in which an especial skill is represented by a ratio between width and distance target at $\mathrm{D}=4.5,5.1$ m. However, our results make us to suggest that once an especial skill is developed, it can be generalized to other distances. This conclusion is different from the results of previous studies on especial skills $(7,9,24)$ and the parameter component of Schema theory [1].

The variability of force is one of the mentioned principles by Schmidt et al. [1] which is about fast movements. As the performer take distance from the target, his targeting performance will be decreased. But it is questioned by recent studies regarding especial skill. Numerous studies show lack of performance coincidence in FT with predicted score. In this study, this lack of coincidence was seen in another distance in addition to the FT (the far distance from the basket, 5.3) in which we keep the index difficulty of FT by manipulating the width of the basket and the performance in that distance has no difference with the FT. These results are considered as a kind of challenge for the principle of variability of force in fast movements. We agree that some factors such as the amount of training and type of training (fixed and variable) can affect the formation of especial skill. In this study, we mentioned another principle factor (index of difficulty) and preserved it. And also, transfer of skill is better when both the size and distance of throw are changed by the same factor (ID is constant) than when only size or distance is changed. We suggest that the widthdistance ratio captures the control strategies employed during practice and thus promotes efficient transfer. This factor can cause presentation of dedicated memory and increase of especial skill in other distances. One of the possible reasons is the visual context which is created in individuals at different distances. It is established at the distance of 4 and 6 because of the similarity of this image. Because of the size variation in target size and distance, the index of difficulty is same as the FT. According to Tau law, the emission of light from two corners of an object to our eyes forms an angle named theta. This angle remains fixed by keeping the ratio between the width of target and distance fix. We keep this angle fix by manipulating the width of the target. The results confirmed the hypothesis of parameter learning for the special skill which is the sign of the difference in predicated and real performance in FT. But our question was about the difference of players' performance in the shot state toward the standard and manipulated basket from $5.3 \mathrm{~m}$ distance in which the index of difficulty of the basketball free throw was kept. According to the view of dynamic systems, it can be said that stability can be accomplished by the transition from a chaotic situation to a stable state and frequent training in FT. This state is created by stabilizing environmental conditions such as distance from the target and visual context. The ratio of the distance from the target to the width of target creates a stable pattern in mind violation that causes turbulence, thus keeping it in any condition followed by a kind of stability [24, 25].

\section{Conclusion}

Envisaged that it is possible that training from a specific distance and throw toward a basket with specific 
size over the years will lead to the formation of especial skill and dedicated memory within which a ratio of distance from target and width of target is stabilized in the mind, in other words, the mind uses index of difficulty as a factor for stabilizing the learning and essential sign for creating dedicated memory.

Finally, it can be suggested that paying attention to parameter learning hypothesis, the visual context in

\section{References}

1. Schmidt RA, Lee TD. Motor control and learning: A behavioral emphasis (4th ed). Skill learning. Psychological review. Champaign IL: Human Kinetics; 2005.

2. Bernstein NA. On dexterity and its development. In: Latash ML, Turvey MT. (Eds). Dexterity and it's development. Mahwah N J. Erlbaum Publishers; 1996. P. 1-244.

3. Raibert MH. Motor control and learning by the statespace model (Tech. Rep. No. AI-TR-439). Cambridge: Massachusetts Institute of Technology, Artificial Intelligence Laboratory; 1977.

4. Adams Jack A. A closed-loop theory of motor learning. Journal of motor behavior, 1971; 3.2: 111-150.]

5. Okazaki V, Lamas L, Okazaki F, Rodacki A. Efeito da distância sobre o arremesso no basquetebol desempenhado por crianças [Effect of distance on the pitch in basketball played by children]. Motricidade. 2013;9(2):62-73. (In Portuguese)

6. Keetch KM, Lee TD, Schmidt RA. Especial skills: Specificity embedded within generality. Journal of Sport \& Exercise Psychology, 2008; 30: 723-736.

7. Breslin Gavin. An especial skill: Support for a learned parameters hypothesis. Acta psychologica, 2010, 134(1): 5560 .

8. Nabavi-Nik Mahdi, Taheri Hamid-Reza, Moghadam Amir. Massive amount of practice and special memory representations, "Special Motor Program Hypothesis". Iranian journal of Health \& Physical Activity, 2011; 2: 25-33.

9. Keetch Katherine M. Especial skills: their emergence with massive amounts of practice. Journal of experimental psychology: human perception and performance, 2005; 31(5): 970-980?

10.Breslin Gavin, Schmidt Richard A, Lee Timothy D. 19 Especial skills. Skill Acquisition in Sport: Research, Theory and Practice, 2012; 6: 330-337.

11.Davies Graham M, Thomson Donald M. Memory in context: Context in memory. John Wiley \& Sons; 1988.

12.Kelso JA. Phase transitions and critical behavior in human bimanual coordination. American Journal of PhysiologyRegulatory, Integrative and Comparative Physiology, 1984; 246(6): R1000-R1004.

13.Turvey MT, Fitch HL, Tuller B. Part V: degrees of freedom, coordinative structures and tuning. In: Kelso, J.A.S. (Ed). Human Motor Behavior: An introduction. New Jersey: Erlbaum; 1978.

14.Thelen Esther, Smith Linda B. A dynamic systems approach to the development of cognition and action. MIT press; 1996 . the representation of the dedicated memory of index of difficulty and also preserving it can lead to the representation of dedicated memory. It can be used as a new factor for representation of memory.

\section{Conflict of interests}

The authors declare that there is no conflict of interests.

15.Taheri HR, Talebi R, Kheir Andish A. The effect of manipulated of the distance and width of target on learning in basketball free threw: according to index of difficult. Motor behavior, 2015;7 (19):50-33.

16.Hacker W. Anforderungen a Regulation und Zeitbedarf bei gef"uhrten Bewegungen: Zur G"ultigkeit des DerwortVon Weizs"ackerschen Gesetzes der konstanten Figurzeit [Accuracy constraints and required duration for guided movements: The validity of Derwort-Von Weizs'acker's isochrony principle]. Zeitschrift fü Psychologie, 1974;182: 307-337. (In German)

17.Kwon OS, Zelaznik HN, Chiu G, Pizlo Z. Human motor transfer is determined by the scaling of size and accuracy of movement. J Motor Behav. 2010;43(1):15-26.

18.Breslin Gavin. Constant or variable practice: Recreating the especial skill effect. Acta psychologica, 2012; 140(2): 154157 .

19.Newell Karl M. Schema theory: Retrospectives and prospectives. Research quarterly for exercise and sport, 2003; 74(4): 383-388.

20.Stöckel Tino, Breslin Gavin. The influence of visual contextual information on the emergence of the especial skill in basketball. Journal of Sport and Exercise Psychology, 2013; 35(5): 536-541.

21.Proteau Luc. On the specificity of learning and the role of visual information for movement control. Advances in psychology, 1992; 85: 67-103.

22.Proteau Luc, Marteniuk Ronald G, Lévesque Line. A sensorimotor basis for motor learning: Evidence indicating specificity of practice. The Quarterly Journal of Experimental Psychology, 1992; 44(3): 557-575.

23.Park Jin-Hoon, Shea Charles H. Sequence learning: Response structure and effector transfer. The Quarterly Journal of Experimental Psychology Section A, 2005; 58(3): 387-419.]

24.Simons JP, Wilson J, Wilson G, Theall S. Challenges to cognitive bases for and especial motor skill emerging at the regulation baseball pitching distance. Research Quarterly for Exercise and Sport, 2009;80(3):469-479.

25.Nabavi Nik Mehdi, Abbas Bahram, Hamid Reza Taheri, Mahnaz Zolghadr. Investigation of visual context hypothesis on especial skills in the favorite location of experienced players. Pizhūhish dar mudīriyyat-i varzish $\bar{\imath}$ va raftār $-i$ harkatī, 2016;6(11): 20-28.' (In Arabic)

26.Khan MA, Franks IM. The utilization of visual feedback in the acquisition of motor skills. In: A. M. Williams, \& N. J. Hodges (Eds.), Skill Acquisition In Sport Research: Theory and Practice. London: Routledge; 2004. P. 45-62. 


\section{Information about the authors:}

Roohollah Talebi (Corresponding author) ; PHD student of motor behavior; http//orcid.org/0000-0002-7593-943X; rohollah.talebi@yahoo.com; Ferdowsi University of Mashhad; Daneshgah square, vakil abad street Mashhad, Iran.

Hamid Reza Taheri; Associate Professor of Physical Education Faculty and Sports Sciences; http://orcid.org/0000-0002-3949-9844; hamidtaherii@gmail.com; Ferdowsi University of Mashhad; Daneshgah square, vakil abad street Mashhad, Iran.

Mahdi Sohrabi; Associate Professor of physical education faculty and sports sciences; http://orcid.org/0000-0003-1656-7661; sohrabi@ferdowsi.um.ac.ir; Ferdowsi University of Mashhad; Daneshgah square, vakil abad street Mashhad, Iran.

Cite this article as: Roohollah Talebi, Hamid Reza Taheri, Mahdi Sohrabi. Index of difficulty which is an effective factor on especial skill formation in basketball free throw: index of learning difficulty hypothesis. Physical education of students, 2018;22(2):83-90. doi:10.15561/20755279.2018.0205

The electronic version of this article is the complete one and can be found online at: http://www.sportedu.org.ua/index.php/PES/issue/archive

This is an Open Access article distributed under the terms of the Creative Commons Attribution License, which permits unrestricted use, distribution, and reproduction in any medium, provided the original work is properly cited (http://creativecommons.org/licenses/by/4.0/deed.en).

Received: 29.12.2017

Accepted: 19.01.2018; Published: 27.04.2018 